\title{
Stability Analysis for Viral Infection Model with Multitarget Cells, Beddington-DeAngelis Functional Response, and Humoral Immunity
}

\author{
Xinxin Tian ${ }^{1}$ and Jinliang Wang ${ }^{1,2}$ \\ ${ }^{1}$ School of Mathematical Science, Heilongjiang University, Harbin 150080, China \\ ${ }^{2}$ School of Mathematics and Statistics, Southwest University, Chongqing 400715, China \\ Correspondence should be addressed to Jinliang Wang; jinliangwang@aliyun.com
}

Received 13 July 2014; Revised 8 September 2014; Accepted 10 September 2014

Academic Editor: Zhen Jin

Copyright (C) 2015 X. Tian and J. Wang. This is an open access article distributed under the Creative Commons Attribution License, which permits unrestricted use, distribution, and reproduction in any medium, provided the original work is properly cited.

We formulate a $(2 n+2)$-dimensional viral infection model with humoral immunity, $n$ classes of uninfected target cells and $n$ classes of infected cells. The incidence rate of infection is given by nonlinear incidence rate, Beddington-DeAngelis functional response. The model admits discrete time delays describing the time needed for infection of uninfected target cells and virus replication. By constructing suitable Lyapunov functionals, we establish that the global dynamics are determined by two sharp threshold parameters: $\mathfrak{R}_{0}$ and $\mathfrak{R}_{1}$. Namely, a typical two-threshold scenario is shown. If $\mathfrak{R}_{0} \leq 1$, the infection-free equilibrium $P_{0}$ is globally asymptotically stable, and the viruses are cleared. If $\Re_{1} \leq 1<\mathfrak{R}_{0}$, the immune-free equilibrium $P_{1}$ is globally asymptotically stable, and the infection becomes chronic but with no persistent antibody immune response. If $\Re_{1}>1$, the endemic equilibrium $P_{2}$ is globally asymptotically stable, and the infection is chronic with persistent antibody immune response.

\section{Introduction}

In the study of mathematical models of infectious diseases in vivo, it is an important problem to predict whether the infection disappears or the pathogens and the immune system persist. In general, the mathematical models of infectious diseases in vivo have a general immune response. For instance, CTLs can kill infected cells or they can secrete soluble factors, which can inhibit viral replication. Another immune response to viral infection is antibody immune response (humoral immunity), which is widely developed to analyze the dynamics of infections agents such as HIV and malaria (see, e.g., $[1,2]$ ).

The basic viral infection model with antibody immunity response studied by Kajiwara and Sasaki [3] takes the following four-dimensional nonlinear differential equations:

$$
\begin{gathered}
x^{\prime}(t)=\lambda-d x(t)-\beta x(t) v(t), \\
y^{\prime}(t)=\beta x(t) v(t)-p y(t),
\end{gathered}
$$

$$
\begin{gathered}
v^{\prime}(t)=k y(t)-\mu v(t)-q v(t) w(t), \\
w^{\prime}(t)=g v(t) w(t)-h w(t),
\end{gathered}
$$

where the uninfected $\mathrm{CD} 4^{+} \mathrm{T}$ cells, $x(t)$, are produced at a constant rate of $\lambda$, die at a rate of $d$, and become infected at a rate of $\beta x v$. The infected cells, $y(t)$, are produced at a rate of $\beta x v$. $\beta$ and $p$ are the infection rate and death rate of infected cells, respectively. Free virus particles, $v(t)$, are released from infected cells at a rate of $k . \mu$ is the death rate of virus. $w(t)$ denotes the density of the antibody immune response (B cells). $g$ and $\mu$ are the birth rate and death rate of B cells. $q$ is the B cells neutralization rate.

In [3], Kajiwara and Sasaki studied the local stability of the equilibria of system (1). But local stability is not equivalent to global stability. The question of global stability in population models is a very interesting mathematical problem. Many authors have studied the global stability of virus dynamics models without delay using the second Lyapunov method. The Lyapunov function candidate for population biology 
models is the Volterra-type function which was applied in literatures [4-9] to prove global stability of the steady states of viral infections models with discrete intracellular delay and distributed delay. But they all ignore the antibody immune response. In [8], Wang and Zou investigated the dynamical behavior of in-host viral models with humoral immunity and intracellular delays. By means of constructing suitable Lyapunov functionals and Lasalle invariance principles, they established the global dynamics by two sharp thresholds.

Most of the existing delayed HIV infection models are based on the assumption that the virus attacks one class of target cells, $\mathrm{CD}^{+}{ }^{+} \mathrm{T}$ cells. In $[10,11]$, an HIV model with two target cells, $\mathrm{CD}^{+}{ }^{+} \mathrm{T}$ cells and macrophages, has been proposed. Recently, a series of work [12-17] has been done for HIV models with two target cells with discrete-time and distributed delays. Lyapunov functionals are proved to be effective tool to establish the global asymptotic stability of their steady states [18-20].

Let $x_{i}(t)$ and $y_{i}(t)$ be the populations of the uninfected target cells and infected cells of class $i$, respectively, and let $v(t)$ be the population of the virus particles. The basic virus infection model with multitarget cells proposed by Elaiw [15] takes the following form of ordinary differential equations:

$$
\begin{gathered}
\frac{d x_{i}(t)}{d t}=\lambda_{i}-d_{i} x_{i}(t)-\beta_{i} x_{i}(t) v(t), \\
\frac{d y_{i}(t)}{d t}=\beta_{i} x_{i}(t) v(t)-p_{i} y_{i}(t), \\
\frac{d v(t)}{d t}=\sum_{i=1}^{n} k_{i} y_{i}(t)-c v(t),
\end{gathered}
$$

where, for $i=1,2, \ldots, n, \lambda_{i}, d_{i}$, and $\beta_{i}$ represent the rates of which new target cells are generated, the death rate constants, and the infection rate constants, respectively. The infected cells die with rate constants $p_{i}$. The virus particles are produced by the infected cells with rate constants $k_{i}$ and are cleared with rate constant $c$.

From system (2), the rate of infection of these viral dynamics models is assumed to be bilinear in the virus $v$ and the uninfected CD $4^{+} \mathrm{T}$ cells $x_{i}$. However, the actual incidence rate is probably not linear over the entire range of $v$ and $x_{i}$. Thus, it is reasonable to assume that the infection rate of viral infection model is given by the Beddington-DeAngelis functional response, $\beta_{i} x_{i} v /\left(1+a_{i} x_{i}+b_{i} v\right)$, where $a_{i}, b_{i} \geq 0$ $[7,21]$. Time delays are intrinsic to the viral infection and replication processes, since antigenic stimulation generating immune response may need a period of time. Let parameter $\tau$ account for the time between viral entry into a target cell and the production of new virus particles. The recruitment of virus-producing cells at time $t$ is given by the number of cells that were newly infected at time $t-\tau$ and are still alive at time $t$. Let $m_{i}$ be a constant rate for infected but not yet virusproducing cells; the probability of surviving the time period from $t-\tau$ to $t$ is $e^{-m_{i} \tau}$.
Combining model (1), model (2), and the preceding assumptions above, we arrive at the following viral infection model:

$$
\begin{gathered}
x_{i}^{\prime}(t)=\lambda_{i}-d_{i} x_{i}(t)-\frac{\beta_{i} x_{i}(t) v(t)}{1+a_{i} x_{i}(t)+b_{i} v(t)}, \\
y_{i}^{\prime}(t)=\frac{\beta_{i} e^{-m_{i} \tau} x_{i}(t-\tau) v(t-\tau)}{1+a_{i} x_{i}(t-\tau)+b_{i} v(t-\tau)}-p_{i} y_{i}(t), \\
v^{\prime}(t)=\sum_{i=1}^{n} k_{i} y_{i}(t)-\mu v(t)-q v(t) w(t), \\
w^{\prime}(t)=g v(t) w(t)-h w(t) .
\end{gathered}
$$

Here the state variables $x_{i}, y_{i}, v$, and $w$ and parameters $\lambda_{i}, d_{i}, \beta_{i}, m_{i}, p_{i}, k_{i}, \mu, q, g$, and $h$ have the same biological meanings as in the systems (1) and (2). $a_{i}$ and $b_{i}$ are constant.

For system (3), we derive the basic reproductive number $\mathfrak{R}_{0}$ and antibody immune activation number $\mathfrak{R}_{1}$ and show that $\mathfrak{R}_{0}$ and $\mathfrak{R}_{1}$ completely determine the global dynamics. The global stability result for the equilibria is new for in-host models with Beddington-DeAngelis functional response, antibody immune response, and intracellular delays. Our proof utilizes a global Lyapunov functional that is motivated by the earlier works mentioned above. The global stability of equilibria rules out any possibility for Hopf bifurcations and existence of sustained oscillations.

The paper is organized as follows. Section 2 is devoted to showing the positivity and ultimate boundedness of the solutions for (1) under suitable initial conditions. Then, we introduce threshold parameter, the basic reproduction number for viral infection $\mathfrak{R}_{0}$, antibody immune response reproduction number $\mathfrak{R}_{1}$, and three possible equilibria for (3). In Section 3, we establish global asymptotic stability of infection-free equilibrium by constructing Lyapunov functional. For a special case $i=1$, we give the proof of global stability of equilibria for a 4-dimensional model in Section 4. We provide some numerical simulations which confirm our analysis in Section 5. In the last section, we offer a brief discussion.

\section{Preliminaries}

The initial conditions for system (3) take the following form:

$$
\begin{array}{r}
x_{i}(\theta)=\phi_{i}(\theta), \quad y_{i}(\theta)=\psi_{i}(\theta), \\
v(\theta)=\chi(\theta), \quad w(\theta)=\varphi(\theta), \\
\phi_{i}(\theta), \psi_{i}(\theta), \chi(\theta), \varphi(\theta) \geq 0, \quad \theta \in[-\tau, 0], \\
\phi_{i}(0), \psi_{i}(0), \chi(0), \varphi(0)>0, \quad i=1,2,3, \ldots, n, \\
\text { where }\left(\phi_{i}(\theta), \psi_{i}(\theta), \chi(\theta), \psi(\theta)\right) \in \mathscr{C}\left([-\tau, 0], R_{+}^{2 n+2}\right) .
\end{array}
$$

2.1. Nonnegativity and Boundedness of Solutions. In the following, we establish the nonnegativity and boundedness of solutions of (3). 
Theorem 1. Let $\left(x_{i}(t), y_{i}(t), v(t), w(t)\right)$ be any solution of system (3) satisfying the initial conditions (4); then $x_{i}(t), y_{i}(t)$, $v(t)$, and $w(t)$ are all nonnegative for $t>0$ and are ultimately bounded.

Proof. First, for $i=1,2, \ldots, n$, we prove that $x_{i}(t)>0$ for all $t \geq 0$. Assume the contrary and let $t_{0}>0$ be such that $x_{i}\left(t_{0}\right)<0$. Set $t_{01}=\inf \left\{0<t<t_{0}: x_{i}(t)<0\right\}$. Then $x_{i}\left(t_{01}\right)=0$, and from the first equation of system (3) we have $x_{i}^{\prime}\left(t_{01}\right)=\lambda_{i}>0$. Hence $x(t)<0$ for $t \epsilon\left(t_{01}-\epsilon, t_{01}\right)$ and $\epsilon>0$ sufficiently small. This contradicts $x(t) \geq 0$ for $t \in\left(0, t_{01}\right]$. It follows that, for $i=1,2, \ldots, n, x_{i}(t)>0$ for $t \geq 0$.

Furthermore, if there exists $t_{1}>0$, such that $y_{i}\left(t_{1}\right)=0$, and $y_{i}(t)>0$ for all $t \in\left(0, t_{1}\right)$, then by the second equation of system (3), we can easily obtain $y_{i}^{\prime}(t) \geq-p_{i} y_{i}(t)$. And it follows that $y_{i}(t) \geq e^{-p_{i} t}$ for all $t \geq 0$. This is a contradiction to the assumption of $y_{i}\left(t_{1}\right)=0$. Hence, $y_{i}(t)>0$, for all $t \geq 0$.

Finally, if there exists $t_{2}>0$, such that $v\left(t_{2}\right)=0$, and $v(t)>0$, for $t \in\left(0, t_{2}\right)$, then by the forth equation of system (3), we have $v^{\prime}(t) \geq-u v(t)-q v(t) w(t)$. And it follows that $v(t) \geq e^{-(u+q w(t))}$ for all $t \geq 0$. This contradicts with the assumption of $v\left(t_{2}\right)=0$. Hence, $v(t)>0$, for all $t \geq 0$. Similarly, we have $w(t)>0$, for $t \in\left(0, t_{3}\right)$.

Next we show that the solution is ultimately bounded. It follows from the first equation of system (3) that

$$
x_{i}^{\prime}(t) \leq \lambda_{i}-d_{i} x_{i}(t) .
$$

Thus $\limsup _{t \rightarrow \infty} x_{i}(t) \leq \lambda_{i} / d_{i}$ and $x_{i}(t)$ is ultimately bounded. Let

$$
X_{i}=e^{-m_{i} \tau} x_{i}(t-\tau)+y_{i}(t)
$$

then

$$
X_{i}^{\prime}(t) \leq \lambda_{i} e^{-m_{i} \tau}-\sigma_{i} X_{i}(t),
$$

where $\sigma_{i}=\min \left\{d_{i}, p_{i}\right\}$. It follows that $\limsup _{t \rightarrow \infty} X_{i} \leq$ $M_{1}$, where $M_{1}=\lambda_{i} e^{-m_{i} \tau} / \sigma_{i}$. This in turn implies, by the nonnegativity of $x_{i}(t)$ and $y_{i}(t)$, that $\limsup _{t \rightarrow \infty} y_{i} \leq M_{1}$ and $y_{i}(t)$ is ultimately bounded. On the other hand, from the third equation of system (3) we have

$$
v^{\prime}(t) \leq \sum_{i=1}^{n} k_{i} M_{1}-u v-q v w
$$

then $\lim \sup _{t \rightarrow \infty} v(t) \leq M_{2}$, where $M_{2}=\sum_{i=1}^{n}\left(k_{i} M_{1} / u\right)$ and $v(t)$ is ultimately bounded. Let $V=v+w$; then

$$
V^{\prime} \leq \sum_{i=1}^{n} k_{i} y_{i}-u v-\left(d M_{2}+h\right) w,
$$

where $d=\min \{q, g\}$; then let $m=\min \left\{u, d M_{2}+h\right\}$; then

$$
V^{\prime} \leq \sum_{i=1}^{n} k_{i} y_{i}-m V
$$

It follows that $\limsup _{t \rightarrow \infty} V \leq M_{3}$, where $M_{3}=\left(\sum_{i=1}^{n} k_{i} y_{i}\right) /$ $m$. This in turn implies, by the nonnegativity of $v(t)$ and $w(t)$, that $\limsup _{t \rightarrow \infty} w(t) \leq M_{3}$ and $w(t)$ is ultimately bounded.
2.2. Reproduction Numbers and Steady States. The basic reproductive number of the virus for system (3) is

$$
\mathfrak{R}_{0}=\sum_{i=1}^{n} R_{i}
$$

where $R_{i}=\lambda_{i} \beta_{i} k_{i} e^{-m_{i} \tau} /\left(d_{i} p_{i} \mu+a_{i} p_{i} \mu \lambda_{i}\right)$. We will know that if $\Re_{0} \leq 1$, then infection-free equilibrium $P_{0}=\left(x_{i}^{0}, 0,0,0\right)$, $x_{i}^{0}=\lambda_{i} / d_{i}$, is the unique steady state. If $\mathfrak{R}_{0}>1$, then in addition to the uninfected steady state there exists an immune-free equilibrium $P_{1}=\left(x_{i}^{1}, y_{i}^{1}, v^{1}, 0\right)$, corresponding to the situation that infection becomes chronic but with no persistent antibody immune response, which is given by the following expressions:

$$
\begin{gathered}
x_{i}^{1}=\frac{\sum_{i=1}^{n}\left(\lambda_{i} b_{i} k_{i}-\lambda_{i} k_{i} \beta_{i}\right)+d_{i} p_{i} u e^{m_{i} \tau}+\lambda_{i} k_{i} \beta_{i}}{d_{i}\left(k_{i} \beta_{i}+\sum_{i=1}^{n} b_{i} d_{i} k_{i}-a_{i} p_{i} \mu e^{m_{i} \tau}\right)}, \\
y_{i}^{1}=\frac{\sum_{i=1}^{n} \beta_{i} e^{-m_{i} \tau} k_{i} \lambda_{i}-d_{i} p_{i} \mu-a_{i} p_{i} \mu \lambda_{i}}{\sum_{i=1}^{n} p_{i} k_{i} d_{i} b_{i}+\beta_{i} k_{i} p_{i}-p_{i}^{2} a_{i} \mu e^{m_{i} \tau}\left(R_{i}-1\right),} \\
v^{1}=\sum_{i=1}^{n} \frac{k_{i}}{\mu}\left(\frac{\sum_{i=1}^{n} \beta_{i} e^{-m_{i} \tau} k_{i} \lambda_{i}-d_{i} p_{i} \mu-a_{i} p_{i} \mu \lambda_{i}}{\sum_{i=1}^{n} p_{i} k_{i} d_{i} b_{i}+\beta_{i} k_{i} p_{i}-p_{i}^{2} a_{i} \mu e^{m_{i} \tau}}\right)\left(R_{i}-1\right) .
\end{gathered}
$$

Now, we introduce an antibody immune response reproduction number:

$$
\begin{aligned}
& \mathfrak{R}_{1} \\
& =\sum_{i=1}^{n} \frac{\mu\left(\sum_{i=1}^{n} p_{i} k_{i} d_{i} b_{i}+\beta_{i} k_{i} p_{i}-p_{i}^{2} a_{i} \mu e^{m_{i} \tau}\right)}{g k_{i}\left(\sum_{i=1}^{n} \beta_{i} e^{-m_{i} \tau} k_{i} \lambda_{i}-d_{i} p_{i} \mu-a_{i} p_{i} \mu \lambda_{i}\right)}\left(g v^{1}-h\right)+1 .
\end{aligned}
$$

Further, if

$$
\mathfrak{R}_{0}>1+\sum_{i=1}^{n} \frac{\mu\left(\sum_{i=1}^{n} p_{i} k_{i} d_{i} b_{i}+\beta_{i} k_{i} p_{i}-p_{i}^{2} a_{i} \mu e^{m_{i} \tau}\right) h}{g k_{i}\left(\sum_{i=1}^{n} \beta_{i} e^{-m_{i} \tau} k_{i} \lambda_{i}-d_{i} p_{i} \mu-a_{i} p_{i} \mu \lambda_{i}\right)},
$$

system (3) also has an endemic equilibrium $P_{2}=\left(x_{i}^{*}, y_{i}^{*}\right.$, $\left.v^{*}, w^{*}\right)$, where

$$
\begin{gathered}
x_{i}^{*}=\left(a_{i} \lambda_{i}-d_{i}-\frac{b_{i} d_{i} h}{g}-\frac{\beta_{i} h}{g}\right. \\
+\left(\left(a_{i} \lambda_{i}-d_{i}-\frac{b_{i} d_{i} h}{g}-\frac{\beta_{i} h}{g}\right)^{2}\right. \\
\left.\left.+4 a_{i} d_{i} \lambda_{i}+\frac{4 \lambda_{i} b_{i} h a_{i} d_{i}}{g}\right)^{1 / 2}\right) \\
\times\left(2 a_{i} d_{i}\right)^{-1}, \quad \\
v^{*}=\frac{h}{g},
\end{gathered}
$$

$$
y_{i}^{*}=\frac{\beta_{i} e^{-m_{i} \tau} x_{i}^{*} v^{*}}{p_{i}\left(1+a_{i} x_{i}^{*}+b_{i} v^{*}\right)}, \quad w^{*}=\sum_{i=1}^{n} \frac{k_{i} g y_{i}^{*}}{q h}-\frac{\mu}{q} .
$$

It follows from

$$
\mathfrak{R}_{1}=\mathfrak{R}_{0}-\sum_{i=1}^{n} \frac{\mu\left(\sum_{i=1}^{n} p_{i} k_{i} d_{i} b_{i}+\beta_{i} k_{i} p_{i}-p_{i}^{2} a_{i} \mu e^{m_{i} \tau}\right) h}{g k_{i}\left(\sum_{i=1}^{n} \beta_{i} e^{-m_{i} \tau} k_{i} \lambda_{i}-d_{i} p_{i} \mu-a_{i} p_{i} \mu \lambda_{i}\right)}
$$


that $\Re_{1}>1$ is equivalent to $g v^{1} / h>1$. The latter $g v^{1} / h$ can be regarded as the immune activation effector. It is reasonable that immune response is activated in the case where $\Re_{1}>1$.

\section{Main Results}

In this section, we consider the global asymptotic stability of the three equilibria. Throughout the paper, for easy notation, we adopt $H(s)=s-1-\ln s \geq H(1)=0$ to simplify many of the expressions which follow.

Theorem 2. Consider system (3) and $\mathfrak{R}_{0}$ is defined by (11). The infection-free equilibrium $P_{0}$ of (3) is globally asymptotically stable if $\mathfrak{R}_{0} \leq 1$.

Proof. Define a Lyapunov functional $L_{0}$ as follows:

$$
\begin{aligned}
L_{0}= & \sum_{i=1}^{n} \frac{x_{i}^{0}}{1+a_{i} x_{i}^{0}} e^{-m_{i} \tau} H\left(\frac{x_{i}}{x_{i}^{0}}\right)+y_{i}+\frac{p_{i}}{k_{i}} v+\frac{p_{i} q}{k_{I} g} w \\
& +\beta_{i} e^{-m_{i} \tau} \int_{t-\tau}^{t} \frac{x_{i}(\theta) v(\theta)}{1+a_{i} x_{i}(\theta)+b_{i} v(\theta)} d \theta .
\end{aligned}
$$

Using $\lambda_{i}=d_{i} x_{i}^{0}$ and calculating the time derivative of $L_{0}$ along (3) yield

$$
\begin{aligned}
L_{0}^{\prime}=\sum_{i=1}^{n}\left[\frac{e^{-m_{i} \tau}}{1+a_{i} x_{i}^{0}}\left(1-\frac{x_{i}^{0}}{x_{i}(t)}\right) x_{i}^{\prime}(t)+y_{i}^{\prime}(t)\right. & \\
& \left.\quad+\frac{\beta_{i} e^{-m_{i} \tau} x_{i} v}{1+a_{i} x_{i}+b_{i} v}-\frac{\beta_{i} e^{-m_{i} \tau} x_{i}(t-\tau) v(t-\tau)}{1+a_{i} x_{i}(t-\tau)+b_{i} v(t-\tau)}\right] \\
+ & \frac{p_{i}}{k_{i}} v^{\prime}(t)+\frac{p_{i} q}{k_{i} g} w^{\prime}(t) \\
=\sum_{i=1}^{n}\left[-\frac{d_{i} e^{-m_{i} \tau}}{x_{i}\left(1+a_{i} x_{i}^{0}\right)}\left(x_{i}-x_{i}^{0}\right)^{2}\right. & \quad \frac{\beta_{i} e^{-m_{i} \tau} x_{i} v}{\left(1+a_{i} x_{i}^{0}\right)\left(1+a_{i} x_{i}+b_{i} v\right)}-\frac{p_{i} \mu}{k_{i}} v-\frac{p_{i} q h}{k_{i} g} w \\
& \left.\quad+\frac{\beta_{i} e^{-m_{i} \tau} x_{i}^{0} v}{\left(1+a_{i} x_{i}^{0}\right)\left(1+a_{i} x_{i}+b_{i} v\right)}+\frac{\beta_{i} e^{-m_{i} \tau} x_{i} v}{1+a_{i} x_{i}+b_{i} v}\right] \\
=\sum_{i=1}^{n}- & \frac{d_{i} e^{-m_{i} \tau}}{x_{i}\left(1+a_{i} x_{i}^{0}\right)}\left(x_{i}-x_{i}^{0}\right)^{2}+\left(R_{0}-1\right) v \\
& -\sum_{i=1}^{n} \frac{b_{i} \beta_{i} e^{-m_{i} \tau} x_{i}^{0}}{\left(1+a_{i} x_{i}^{0}\right)\left(1+a_{i} x_{i}+b_{i} v\right)} v^{2}-\frac{p_{i} q h}{k_{i} g} w .
\end{aligned}
$$

Thus, $\mathfrak{R}_{0} \leq 1$ ensures that $L_{0}^{\prime}<0$ for all $x_{i}>0, y_{i} \geq 0, v>0$, and $w>0$. And $L_{0}^{\prime}=0$ if and only if $x_{i}=x_{i}^{0}, v=w=0$ for $\mathfrak{R}_{0} \leq 1$. It is easy to show that $P_{0}=\left(x_{i}^{0}, 0,0,0\right)$ is the largest invariant set in $\left\{\left(x_{i}, y_{i}, v, w\right) \mid L_{0}^{\prime}=0\right\}$. By LaSalle invariance principle, the equilibrium $P_{0}\left(x_{i}^{0}, 0,0,0\right)$ is globally asymptotically stable. This completes the proof.

Theorem 3. Consider system (3) and $\mathfrak{R}_{0}$ and $\Re_{1}$ are defined by (11) and (13). The immune-free equilibrium $P_{1}$ of (3) is globally asymptotically stable if $\Re_{1} \leq 1<\mathfrak{R}_{0}$.
Proof. Define a Lyapunov functional $L_{1}$ as follows:

$$
\begin{gathered}
L_{1}=\sum_{i=1}^{n}\left[\frac{x_{i}^{1}\left(1+b_{i} v^{1}\right) e^{-m_{i} \tau}}{1+a_{i} x_{i}^{1}+b_{i} v^{1}} H\left(\frac{x_{i}}{x_{i}^{1}}\right)+y_{i} H\left(\frac{y_{i}}{y_{i}^{1}}\right)\right. \\
+p_{i} y_{i}^{1} \int_{t-\tau}^{t} H\left(\frac{x_{i}(\theta) v(\theta)}{1+a_{i} x_{i}(\theta)+b_{i} v(\theta)}\right. \\
\left.\left.\times \frac{1+a_{i} x_{i}^{1}+b_{i} v^{1}}{x_{i}^{1} v^{1}}\right) d \theta\right] \\
+\frac{p_{i} v^{1}}{k_{i}} H\left(\frac{v}{v^{1}}\right)+\frac{p_{i} q}{k_{i} g} w .
\end{gathered}
$$

The derivative of $L_{1}$ is given by

$$
\begin{aligned}
& L_{1}^{\prime}=\sum_{i=1}^{n}\left[\frac{\left(1+b_{i} v^{1}\right) e^{-m_{i} \tau}}{1+a_{i} x_{i}^{1}+b_{i} v^{1}}\left(1-\frac{x_{i}^{1}}{x_{i}}\right) x_{i}^{\prime}+\left(1-\frac{y_{i}^{1}}{y_{i}}\right) y_{i}^{\prime}\right. \\
& +\frac{\beta e^{-m_{i} \tau} x_{i} v}{1+a_{i} x_{i}+b_{i} v}-\frac{\beta_{i} e^{-m_{i} \tau} x_{i}(t-\tau) v(t-\tau)}{1+a_{i} x_{i}(t-\tau)+b_{i} v(t-\tau)} \\
& +p_{i} y_{i}^{1} \ln \frac{x_{i}(t-\tau) v(t-\tau)}{x_{i} v} \\
& \left.\times \frac{1+a_{i} x_{i}+b_{i} v}{1+a_{i} x_{i}(t-\tau)+b_{i} v(t-\tau)}\right] \\
& +\frac{p_{i}}{k_{i}}\left(1-\frac{v^{1}}{v}\right) v^{\prime}+\frac{p_{i} q}{k_{i} g} w^{\prime} \\
& =\sum_{i=1}^{n}\left[-\frac{d_{i}\left(1+b_{i} v^{1}\right) e^{-m_{i} \tau}}{x_{i}\left(1+a_{i} x_{i}^{1}+b_{i} v^{1}\right)}\left(x_{i}-x_{i}^{1}\right)^{2}\right. \\
& +p_{i} y_{i}^{1}\left[1-\frac{x_{i}^{1}\left(1+a_{i} x_{i}+b_{i} v^{1}\right)}{x_{i}\left(1+a_{i} x_{i}^{1}+b_{i} v^{1}\right)}\right. \\
& \left.+\ln \frac{x_{i}^{1}\left(1+a_{i} x_{i}+b_{i} v^{1}\right)}{x_{i}\left(1+a_{i} x_{i}^{1}+b_{i} v^{1}\right)}\right]
\end{aligned}
$$




$$
\begin{gathered}
+p_{i} y_{i}^{1} \\
\times\left[1-\frac{y_{i}^{1}\left(1+a_{i} x_{i}^{1}+b_{i} v^{1}\right) x_{i}(t-\tau) v(t-\tau)}{y_{i} x_{i}^{1} v^{1}\left(1+a_{i} x_{i}(t-\tau)+b_{i} v(t-\tau)\right)}\right. \\
\left.+\ln \frac{y_{i}^{1}\left(1+a_{i} x_{i}^{1}+b_{i} v^{1}\right) x_{i}(t-\tau) v(t-\tau)}{y_{i} x_{i}^{1} v^{1}\left(1+a_{i} x_{i}(t-\tau)+b_{i} v(t-\tau)\right)}\right] \\
+p_{i} y_{i}^{1}\left[1-\frac{v^{1} y_{i}}{y_{i}^{1} v}+\ln \frac{v^{1} y_{i}}{y_{i}^{1} v}\right] \\
+p_{i} y_{i}^{1}\left[1-\frac{1+a_{i} x_{i}+b_{i} v}{1+a_{i} x_{i}+b_{i} v^{1}}+\ln \frac{1+a_{i} x_{i}+b_{i} v}{1+a_{i} x_{i}+b_{i} v^{1}}\right] \\
+\frac{p_{i} q\left(g y_{i}^{1} b_{i}\left(1+a_{i} x_{i}\right)\left(v-v^{1}\right)^{2}\right.}{k_{i} g} w_{i} \\
\left.+\frac{v^{1}\left(1+a_{i} x_{i}+b_{i} v\right)\left(1+a_{i} x_{i}+b_{i} v^{1}\right)}{2}\right]
\end{gathered}
$$

In the above calculations, we used the equations $\lambda_{i}=$ $d_{i} x_{i}^{1}+\left(\beta_{i} x_{i}^{1} v^{1} /\left(1+a_{i} x_{i}^{1}+b_{i} v^{1}\right)\right), \sum_{i=1}^{n} k_{i} y_{i}^{1}=u v^{1}$, and $\beta_{i} e^{-m_{i} \tau} x_{i}^{1} v^{1} /\left(1+a_{i} x_{i}^{1}+b_{i} v^{1}\right)=p_{i} y_{i}^{1}$.

Further, $\mathfrak{R}_{1} \leq 1<\mathfrak{R}_{0}$ is equivalent to $v^{1} \leq h / g$. Hence $L_{1}^{\prime}$ is always nonpositive under the condition $\mathfrak{R}_{1} \leq 1<\mathfrak{R}_{0}$, and $L_{1}^{\prime}=0$ if and only if $x_{i}=x_{i}^{1}, y_{i} / y_{i}^{1}=v / v^{1}$, and $w=0$ for $\mathfrak{R}_{1}<1$ or $x_{i}=x_{i}^{1}, y_{i} / y_{i}^{1}=v / v^{1}$ for $\mathfrak{R}_{1}=1$. Similarly, it follows from LaSalle invariance principle that the immune-free equilibrium $P_{1}$ is globally asymptotically stable. This completes the proof.

Theorem 4. Consider system (3) and $\mathfrak{R}_{1}$ is defined by (13). The endemic equilibrium $P_{2}$ of (3) is globally asymptotically stable if $\Re_{1}>1$.

Proof. Define a Lyapunov functional $L_{2}$ as follows:

$$
\begin{gathered}
L_{2}=\sum_{i=1}^{n}\left[\frac{x_{i}^{*}\left(1+b v_{i}^{*}\right) e^{-m_{i} \tau}}{1+a_{i} x_{i}^{*}+b_{i} v^{*}} H\left(\frac{x_{i}}{x_{i}^{*}}\right)+y_{i}^{*} H\left(\frac{y_{i}}{y_{i}^{*}}\right)\right. \\
+p_{i} y_{i}^{*} \int_{t-\tau}^{t} H\left(\frac{x_{i}(\theta) v(\theta)}{1+a_{i} x_{i}(\theta)+b_{i} v(\theta)}\right. \\
\left.\left.\times \frac{1+a_{i} x_{i}^{*}+b_{i} v^{*}}{x_{i}^{*} v^{*}}\right) d \theta\right] \\
+\frac{p_{i} v^{*}}{k_{i}} H\left(\frac{v}{v^{*}}\right)+\frac{p_{i} q w^{*}}{k_{i} g} H\left(\frac{w}{w^{*}}\right) .
\end{gathered}
$$

The derivative of $L_{2}$ is given by

$$
\begin{aligned}
& L_{2}^{\prime}=\sum_{i=1}^{n}\left[\frac{e^{-m_{i} \tau}\left(1+b_{i} v^{*}\right)}{1+a_{i} x_{i}^{*}+b_{i} v^{*}}\left(1-\frac{x_{i}^{*}}{x_{i}}\right) x_{i}^{\prime}+\left(1-\frac{y_{i}^{*}}{y_{i}}\right) y_{i}^{\prime}\right. \\
& +\frac{\beta_{i} e^{-m_{i} \tau} x_{i} v}{1+a_{i} x_{i}+b_{i} v}-\frac{\beta_{i} e^{-m_{i} \tau} x_{i}(t-\tau) v(t-\tau)}{1+a_{i} x_{i}(t-\tau)+b_{i} v(t-\tau)} \\
& +p_{i} y_{i}^{*} \ln \frac{x_{i}(t-\tau) v(t-\tau)}{x_{i} v} \\
& \left.\times \frac{1+a_{i} x_{i}+b_{i} v}{1+a_{i} x_{i}(t-\tau)+b_{i} v(t-\tau)}\right] \\
& +\frac{p_{i}}{k_{i}}\left(1-\frac{v^{*}}{v}\right) v^{\prime}+\frac{p_{i} q}{k_{i} g}\left(1-\frac{w^{*}}{w}\right) w^{\prime} \\
& =\sum_{i=1}^{n}\left[-\frac{d_{i}\left(1+b_{i} v^{*}\right) e^{-m_{i} \tau}}{x_{i}\left(1+a_{i} x_{i}^{*}+b_{i} v^{*}\right)}\left(x_{i}-x_{i}^{*}\right)^{2}\right] \\
& +\sum_{i=1}^{n} p_{i} y_{i}^{*}\left[1-\frac{x_{i}^{*}\left(1+a_{i} x_{i}+b_{i} v^{*}\right)}{x_{i}\left(1+a_{i} x_{i}^{*}+b_{i} v^{*}\right)}\right. \\
& \left.+\ln \frac{x_{i}^{*}\left(1+a_{i} x_{i}+b_{i} v^{*}\right)}{x_{i}\left(1+a_{i} x_{i}^{*}+b_{i} v^{*}\right)}\right] \\
& +\sum_{i=1}^{n} p_{i} y_{i}^{*}\left[1-\frac{y_{i}^{*}\left(1+a_{i} x_{i}^{*}+b_{i} v^{*}\right) x_{i}(t-\tau) v(t-\tau)}{y_{i} x_{i}^{*} v^{*}\left(1+a_{i} x_{i}(t-\tau)+b_{i} v(t-\tau)\right)}\right. \\
& \left.+\ln \frac{y_{i}^{*}\left(1+a_{i} x_{i}^{*}+b_{i} v^{*}\right) x_{i}(t-\tau) v(t-\tau)}{y_{i} x_{i}^{*} v^{*}\left(1+a_{i} x_{i}(t-\tau)+b_{i} v(t-\tau)\right)}\right] \\
& -\sum_{i=1}^{n} \frac{p y_{i}^{*} b_{i}\left(1+a_{i} x_{i}\right)\left(v-v^{*}\right)^{2}}{v^{*}\left(1+a_{i} x_{i}+b_{i} v\right)\left(1+a_{i} x_{i}+b_{i} v^{*}\right)} \\
& +\sum_{i=1}^{n} p_{i} y_{i}^{*}\left[1-\frac{v^{*} y_{i}}{y_{i}^{*} v}+\ln \frac{v^{*} y_{i}}{y_{i}^{*} v}\right] \\
& +\sum_{i=1}^{n} p_{i} y_{i}^{*}\left[1-\frac{1+a_{i} x_{i}+b_{i} v}{1+a_{i} x_{i}+b_{i} v^{*}}+\ln \frac{1+a_{i} x_{i}+b_{i} v}{1+a_{i} x_{i}+b_{i} v^{*}}\right] \text {. }
\end{aligned}
$$

Therefore, $L_{2}^{\prime} \leq 0$ holds for all $x_{i}, y_{i}, v, w>0$. Thus, the endemic equilibrium $P_{2}$ is stable. And we have $L_{2}^{\prime}=0$ if and only if $x_{i}=x_{i}^{*}, y_{i}=y_{i}^{*}, v=v^{*}$, and $w=w^{*}$ hold. The largest compact invariant set in $\left\{\left(x_{i}, y_{i}, v, w\right) \mid L_{2}^{\prime}=\right.$ $0\}$ is the singleton $\left\{P_{2}\right\}$. Therefore, the endemic equilibrium $P_{2}$ is globally asymptotically stable by the LaSalle invariance principle when $\mathfrak{R}_{1}>1$. This completes the proof. 


\section{Special Case When $i=1$}

For $i=1$, system (3) becomes

$$
\begin{gathered}
x^{\prime}(t)=\lambda-d x(t)-\frac{\beta x(t) v(t)}{1+a x(t)+b v(t)}, \\
y^{\prime}(t)=\frac{\beta e^{-m \tau} x(t-\tau) v(t-\tau)}{1+a x(t-\tau)+b v(t-\tau)}-p y(t), \\
v^{\prime}(t)=k y(t)-\mu v(t)-q v(t) w(t), \\
w^{\prime}(t)=g v(t) w(t)-h w(t) .
\end{gathered}
$$

Here the state variables $x, y, v$, and $w$ and parameters $\lambda, d, \beta$, $a, b, m, p, k, \mu, q, g$, and $h$ have the similar biological meanings as in the system (3).

The basic reproductive number of the virus for system (23) is $\mathfrak{R}_{0}^{1}=\lambda \beta k e^{-m \tau} /(d p \mu+a p \mu \lambda)$. We will know that if $\mathfrak{R}_{0}^{1} \leq 1$, then infection-free equilibrium $P_{0}=\left(x_{0}, 0,0,0\right)$, $x_{0}=\lambda / d$, is the unique steady state; if $\mathfrak{R}_{0}^{1}>1$, then in addition to the uninfected steady state there exists an immune-free equilibrium $E_{1}=\left(x_{1}, y_{1}, v_{1}, 0\right)$, corresponding to the situation that infection becomes chronic but with no persistent antibody immune response, which is given by the following expressions:

$$
\begin{gathered}
x_{1}=\frac{\lambda b k+p u e^{m \tau}}{k \beta+b d k-a p \mu e^{m \tau}} \\
y_{1}=\frac{d p \mu+a p \mu \lambda}{p\left(k \beta+b d k-a p \mu e^{m \tau}\right)}\left(\Re_{0}^{1}-1\right), \\
v_{1}=\frac{k(d p \mu+a p \mu \lambda)}{p \mu\left(k \beta+b d k-a p \mu e^{m \tau}\right)}\left(\Re_{0}^{1}-1\right) .
\end{gathered}
$$

Now, we introduce an antibody immune response reproduction number:

$$
\mathfrak{R}_{1}^{1}=\frac{p \mu\left(k \beta+b d k-a p \mu e^{m \tau}\right)}{g k(d p \mu+a p \mu \lambda)}\left(g v_{1}-h\right)+1 .
$$

Further, if

$$
\mathfrak{R}_{0}^{1}>1+\frac{p \mu\left(k \beta+b d k-a p \mu e^{m \tau}\right) h}{g k(d p \mu+a p \mu \lambda)},
$$

system (3) also has an endemic equilibrium $E_{2}=\left(x_{2}, y_{2}, v_{2}\right.$, $w_{2}$ ), where

$$
\begin{aligned}
& x_{2}=\left(a \lambda-d-\frac{b d h}{g}-\frac{\beta h}{g}\right. \\
&\left.+\sqrt{\left(a \lambda-d-\frac{b d h}{g}-\frac{\beta h}{g}\right)^{2}+4 a d \lambda+\frac{4 \lambda b h a d}{g}}\right) \\
& \times(2 a d)^{-1}, \\
& v_{2}=\frac{h}{g}, \quad y_{2}=\frac{\beta e^{-m \tau} x_{2} v_{2}}{p\left(1+a x_{2}+b v_{2}\right)}, \quad w_{2}=\frac{k g y_{2}}{q h}-\frac{\mu}{q} .
\end{aligned}
$$

It follows from

$$
\mathfrak{R}_{1}^{1}=\mathfrak{R}_{0}^{1}-\frac{p \mu\left(k \beta+b d k-a p \mu e^{m \tau}\right) h}{g k(d p \mu+a p \mu \lambda)}
$$

that $\mathfrak{R}_{1}^{1}>1$ is equivalent to $g v_{1} / h>1$. The latter $g v_{1} / h$ can be regarded as the immune activation effector. It is reasonable that immune response is activated in the case where $\mathfrak{R}_{1}^{1}>1$.

The initial conditions for system (23) take the following form:

$$
\begin{array}{r}
x(\theta)=\phi_{1}(\theta), \quad y(\theta)=\phi_{2}(\theta), \\
v(\theta)=\phi_{3}(\theta), \quad w(\theta)=\phi_{4}(\theta), \\
\phi_{i}(\theta) \geq 0, \quad \theta \in[-\tau, 0], \quad \phi_{i}(0)>0, \quad i=1,2,3,4,
\end{array}
$$

where $\left(\phi_{1}(\theta), \phi_{2}(\theta), \phi_{3}(\theta), \phi_{4}(\theta)\right) \in \mathscr{C}\left([-\tau, 0], R_{+0}^{4}\right)$.

Theorem 5. Consider system (23).

(i) The infection-free equilibrium $E_{0}$ of (23) is globally asymptotically stable if $\Re_{0}^{1} \leq 1$.

(ii) The immune-free equilibrium $E_{1}$ of (23) is globally asymptotically stable if $\Re_{1}^{1} \leq 1<\Re_{0}^{1}$.

(iii) The endemic equilibrium $E_{2}$ of (23) is globally asymptotically stable if $\Re_{1}^{1}>1$.

Proof. Consider the following.

Proof of (i) of Theorem 5. Define a Lyapunov functional $L_{0}$ as follows:

$$
\begin{aligned}
L_{0}= & \frac{x_{0}}{1+a x_{0}} e^{-m \tau} H\left(\frac{x}{x_{0}}\right)+y+\frac{p}{k} v+\frac{p q}{k g} w \\
& +\beta e^{-m \tau} \int_{t-\tau}^{t} \frac{x(\theta) v(\theta)}{1+a x(\theta)+b v(\theta)} d \theta .
\end{aligned}
$$

Using $\lambda=d x_{0}$ and calculating the time derivative of $U_{0}$ along (23), we have

$$
\begin{aligned}
U_{0}^{\prime}= & \frac{e^{-m \tau}}{1+a x_{0}}\left(1-\frac{x_{0}}{x(t)}\right) x^{\prime}(t)+y^{\prime}(t)+\frac{p}{k} v^{\prime}(t) \\
& +\frac{p q}{k g} w^{\prime}(t)+\frac{\beta e^{-m \tau} x v}{1+a x+b v} \\
& -\frac{\beta e^{-m \tau} x(t-\tau) v(t-\tau)}{1+a x(t-\tau)+b v(t-\tau)} \\
= & -\frac{d e^{-m \tau}}{x\left(1+a x_{0}\right)}\left(x-x_{0}\right)^{2}-\frac{\beta e^{-m \tau} x v}{\left(1+a x_{0}\right)(1+a x+b v)} \\
& -\frac{p \mu}{k} v-\frac{p q h}{k g} w+\frac{\beta e^{-m \tau} x_{0} v}{\left(1+a x_{0}\right)(1+a x+b v)} \\
& +\frac{\beta e^{-m \tau} x v}{1+a x+b v}
\end{aligned}
$$




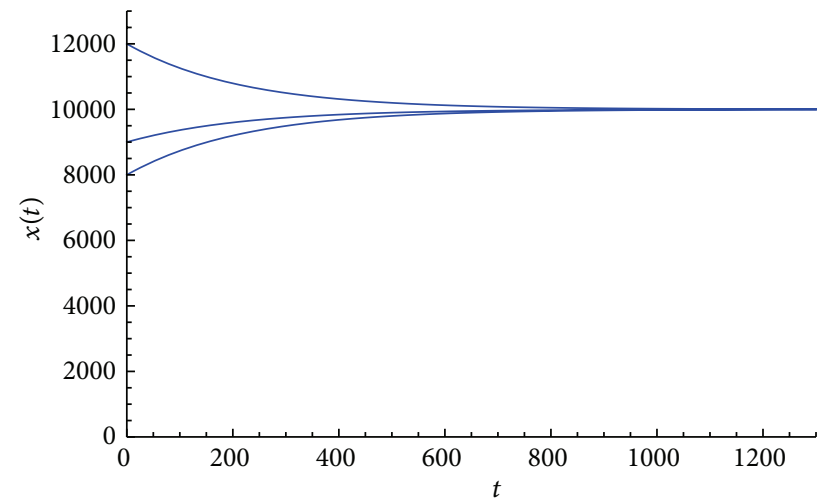

(a)

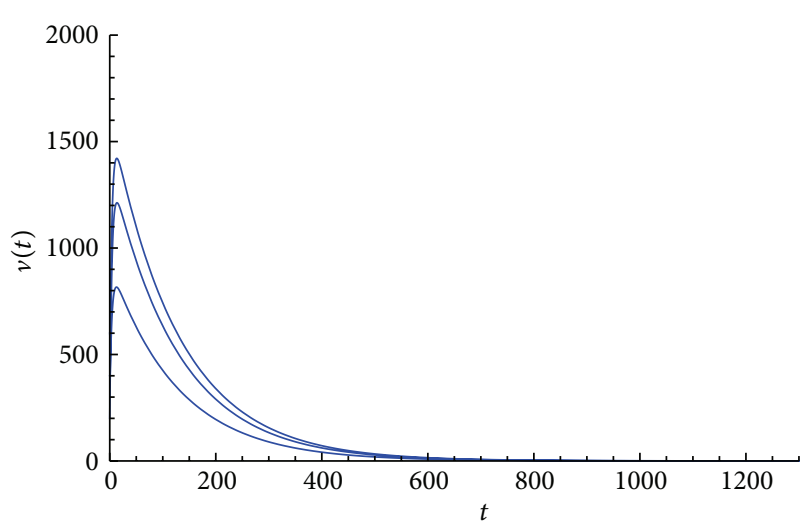

(c)

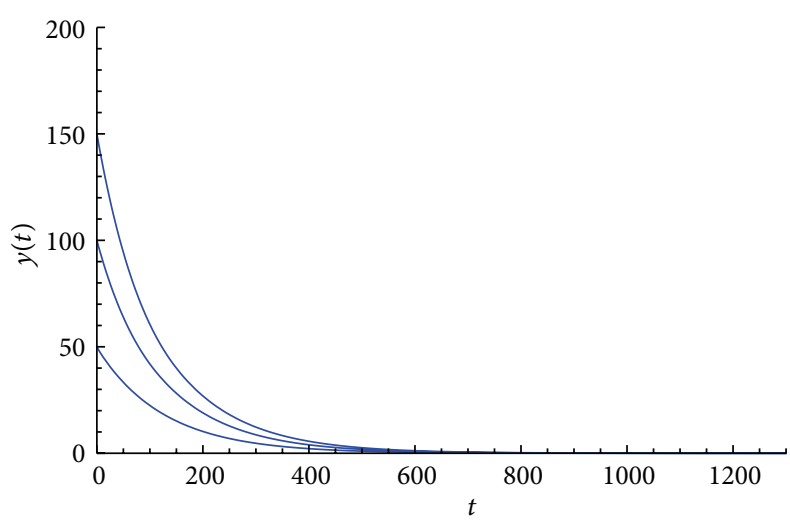

(b)

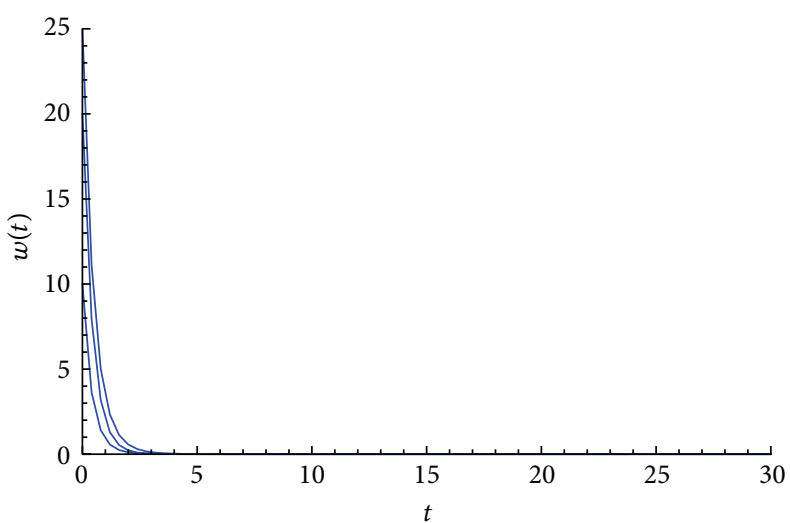

(d)

FIGURE 1: Trajectories of $x(t), x(t), x(t), w(t)$ for $\mathfrak{R}_{0}^{1}=0.215743, \mathfrak{R}_{1}^{1}=0.127203$, and $E_{0}=\left(10^{4}, 0,0,0\right)$ are globally asymptotically stable. $x(t), y(t), v(t)$, and $w(t)$ versus $t$ are illustrated by (a), (b), (c), and (d).

$$
\begin{aligned}
= & -\frac{d e^{-m \tau}}{x\left(1+a x_{0}\right)}\left(x-x_{0}\right)^{2}+\frac{p \mu v(1+a x)}{k(1+a x+b v)}\left(\Re_{0}-1\right) \\
& -\frac{p \mu b}{k(1+a x+b v)} v^{2}-\frac{p q h}{k g} w .
\end{aligned}
$$

Thus, $\mathfrak{R}_{0}^{1} \leq 1$ ensures that $U_{0}^{\prime}<0$ for all $x>0, y \geq 0$, $v>0$, and $w>0$. And $U_{0}^{\prime}=0$ if and only if $x=x_{0}, v=$ $w=0$ for $\mathfrak{R}_{0}^{1} \leq 1$. It is easy to show that $E_{0}\left(x_{0}, 0,0,0\right)$ is the largest invariant set in $\left\{(x, y, v, w) \mid U_{0}^{\prime}=0\right\}$. By LaSalle invariance principle, the equilibrium $E_{0}\left(x_{0}, 0,0,0\right)$ is globally asymptotically stable.

Proof of (ii) of Theorem 5. Define a Lyapunov functional $L_{1}$ as follows:

$$
\begin{aligned}
U_{1}= & \frac{x_{1}\left(1+b v_{1}\right) e^{-m \tau}}{1+a x_{1}+b v_{1}} H\left(\frac{x}{x_{1}}\right) \\
& +y_{1} H\left(\frac{y}{y_{1}}\right)+\frac{p v_{1}}{k} H\left(\frac{v}{v_{1}}\right)+\frac{p q}{k g} w \\
& +p y_{1} \int_{t-\tau}^{t} H\left(\frac{x(\theta) v(\theta)}{1+a x(\theta)+b v(\theta)} \frac{1+a x_{1}+b v_{1}}{x_{1} v_{1}}\right) d \theta .
\end{aligned}
$$

The derivative of $U_{1}$ is given by

$$
\begin{aligned}
U_{1}^{\prime}= & \frac{\left(1+b v_{1}\right) e^{-m \tau}}{1+a x_{1}+b v_{1}}\left(1-\frac{x_{1}}{x}\right) x^{\prime}+\left(1-\frac{y_{1}}{y}\right) y^{\prime} \\
& +\frac{p}{k}\left(1-\frac{v_{1}}{v}\right) v^{\prime}+\frac{p q}{k g} w^{\prime}+\frac{\beta e^{-m \tau} x v}{1+a x+b v} \\
& -\frac{\beta e^{-m \tau} x(t-\tau) v(t-\tau)}{1+a x(t-\tau)+b v(t-\tau)} \\
& +p y_{1} \ln \frac{x(t-\tau) v(t-\tau)}{x v} \frac{1+a x+b v}{1+a x(t-\tau)+b v(t-\tau)} \\
= & -\frac{d\left(1+b v_{1}\right) e^{-m \tau}}{x\left(1+a x_{1}+b v_{1}\right)}\left(x-x_{1}\right)^{2}+\frac{p q\left(g v_{1}-h\right)}{k g} w \\
& +p y_{1}\left[1-\frac{x_{1}\left(1+a x+b v_{1}\right)}{x\left(1+a x_{1}+b v_{1}\right)}+\ln \frac{x\left(1+a x+b v_{1}\right)}{x\left(1+a x_{1}+b v_{1}\right)}\right]
\end{aligned}
$$




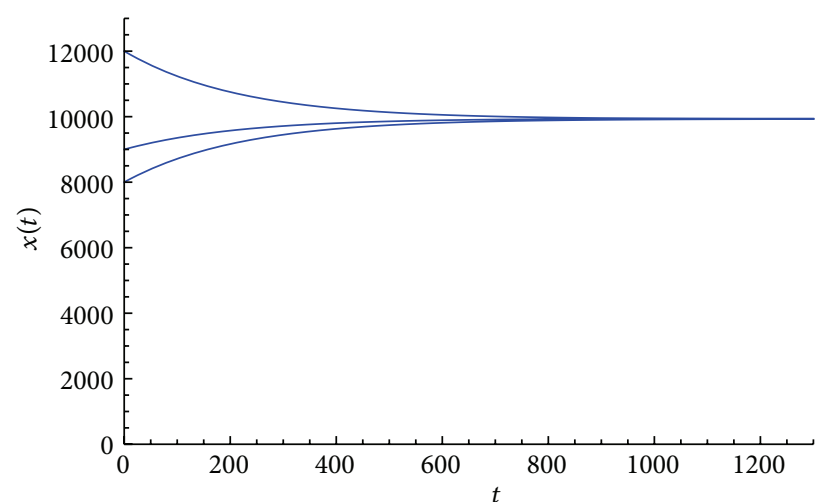

(a)

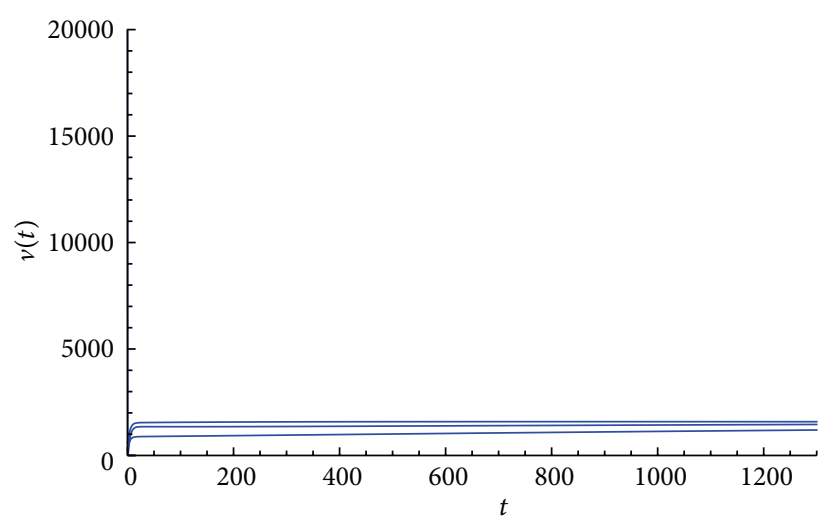

(c)

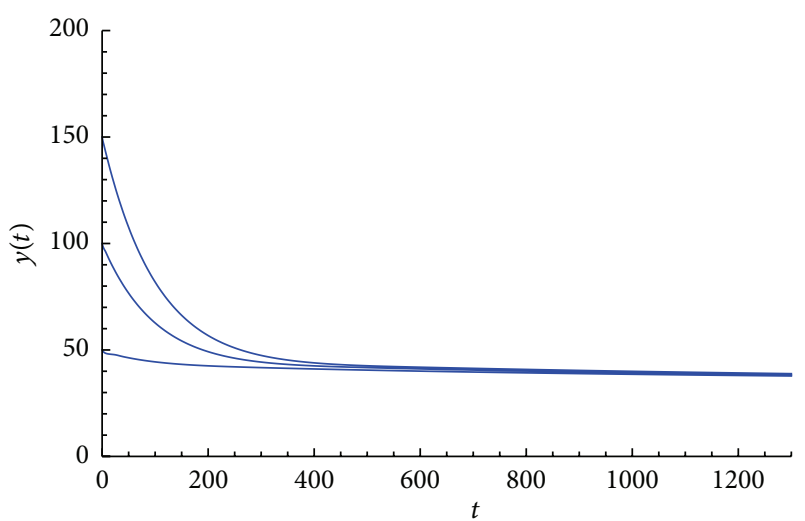

(b)

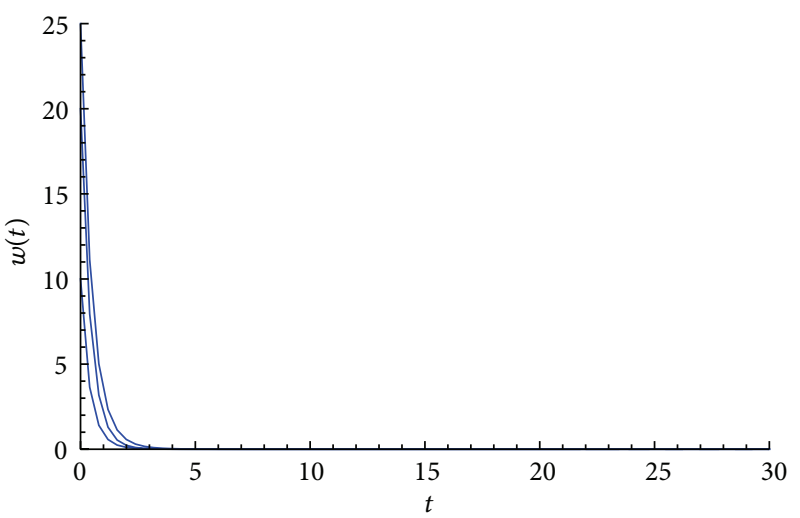

(d)

Figure 2: Trajectories of $x(t), y(t), v(t), w(t)$ for $\mathfrak{R}_{0}^{1}=1.07872, \mathfrak{R}_{1}^{1}=0.982187$, and $E_{1}=(9924.5,34.7292,1576.57,0)$ are globally asymptotically stable. $x(t), y(t), v(t)$, and $w(t)$ versus $t$ are illustrated by (a), (b), (c), and (d).

$$
\begin{array}{r}
+p y_{1}\left[1-\frac{y_{1}\left(1+a x_{1}+b v_{1}\right) x(t-\tau) v(t-\tau)}{y x_{1} v_{1}(1+a x(t-\tau)+b v(t-\tau))}\right. \\
\left.+\ln \frac{y_{1}\left(1+a x_{1}+b v_{1}\right) x(t-\tau) v(t-\tau)}{y x_{1} v_{1}(1+a x(t-\tau)+b v(t-\tau))}\right] \\
+p y_{1}\left[1-\frac{v_{1} y}{y_{1} v}+\ln \frac{v_{1} y}{y_{1} v}\right] \\
+p y_{1}\left[1-\frac{1+a x+b v}{1+a x+b v_{1}}+\ln \frac{1+a x+b v}{1+a x+b v_{1}}\right] \\
-\frac{p y_{1} b(1+a x)\left(v-v_{1}\right)^{2}}{v_{1}(1+a x+b v)\left(1+a x+b v_{1}\right)} .
\end{array}
$$

In the above calculations, we used the equations $\lambda=d x_{1}+$ $\left(\beta x_{1} v_{1} /\left(1+a x_{1}+b v_{1}\right)\right), k y_{1}=u v_{1}$, and $\beta e^{-m \tau} x_{1} v_{1} /\left(1+a x_{1}+\right.$ $\left.b v_{1}\right)=p y_{1}$.

Further, $\mathfrak{R}_{1}^{1} \leq 1<\mathfrak{R}_{0}^{1}$ is equivalent to $v_{1} \leq h / g$. Hence $U_{1}^{\prime}$ is always nonpositive under the condition $\mathfrak{R}_{1}^{1} \leq 1<\mathfrak{R}_{0}^{1}$, and $U_{1}^{\prime}=0$ if and only if $x=x_{1}, y / y_{1}=v / v_{1}$, and $w=0$ for $\mathfrak{R}_{1}^{1}<1$ or $x=x_{1}, y / y_{1}=v / v_{1}$ for $\mathfrak{R}_{1}^{1}=1$. Similarly, it follows from LaSalle invariance principle that the immunefree equilibrium $E_{1}$ is globally asymptotically stable.

Proof of (iii) of Theorem 5. Define a Lyapunov functional $U_{2}$ as follows:

$$
\begin{aligned}
U_{2}= & \frac{x_{2}\left(1+b v_{2}\right) e^{-m \tau}}{1+a x_{2}+b v_{2}} H\left(\frac{x}{x_{2}}\right)+y_{2} H\left(\frac{y}{y_{2}}\right) \\
& +\frac{p v_{2}}{k} H\left(\frac{v}{v_{2}}\right)+\frac{p q w_{2}}{k g} H\left(\frac{w}{w_{2}}\right) \\
& +p y_{2} \int_{t-\tau}^{t} H\left(\frac{x(\theta) v(\theta)}{1+a x(\theta)+b v(\theta)} \frac{1+a x_{2}+b v_{2}}{x_{2} v_{2}}\right) d \theta .
\end{aligned}
$$

The derivative of $U_{2}$ is given by

$$
\begin{aligned}
U_{2}^{\prime}= & \frac{e^{-m \tau}\left(1+b v_{2}\right)}{1+a x_{2}+b v_{2}}\left(1-\frac{x_{2}}{x}\right) x^{\prime}+\left(1-\frac{y_{2}}{y}\right) y^{\prime} \\
& +\frac{p}{k}\left(1-\frac{v_{2}}{v}\right) v^{\prime}+\frac{p q}{k g}\left(1-\frac{w_{2}}{w}\right) w^{\prime}
\end{aligned}
$$




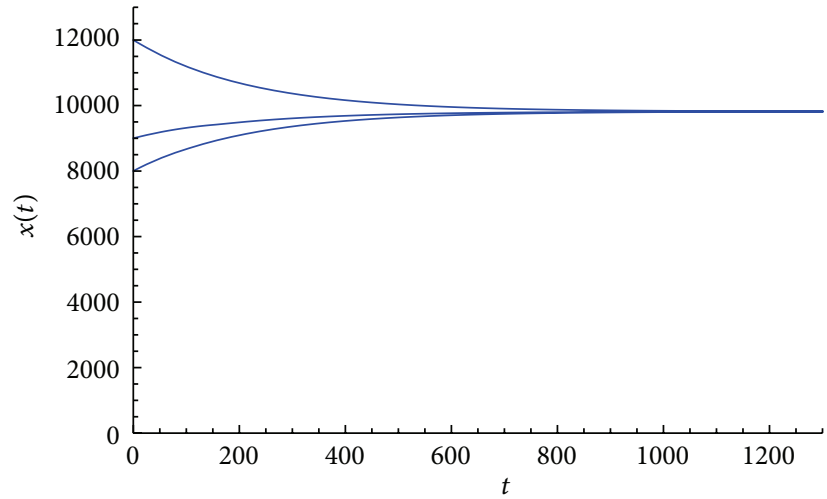

(a)

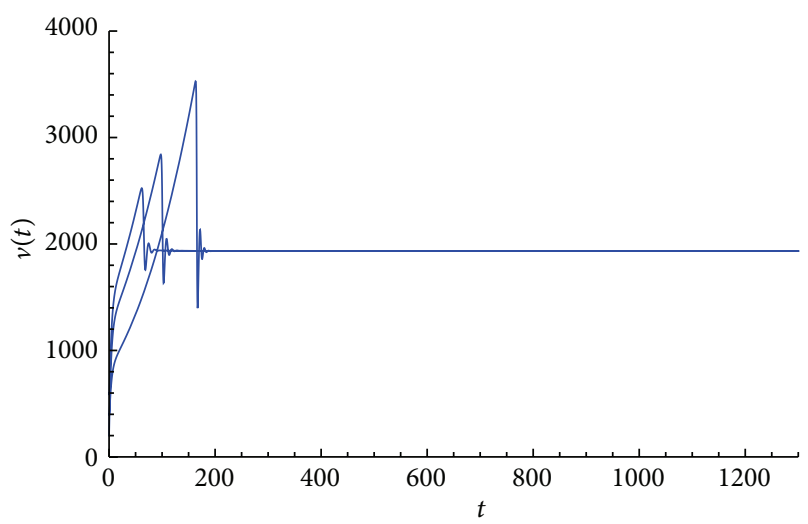

(c)

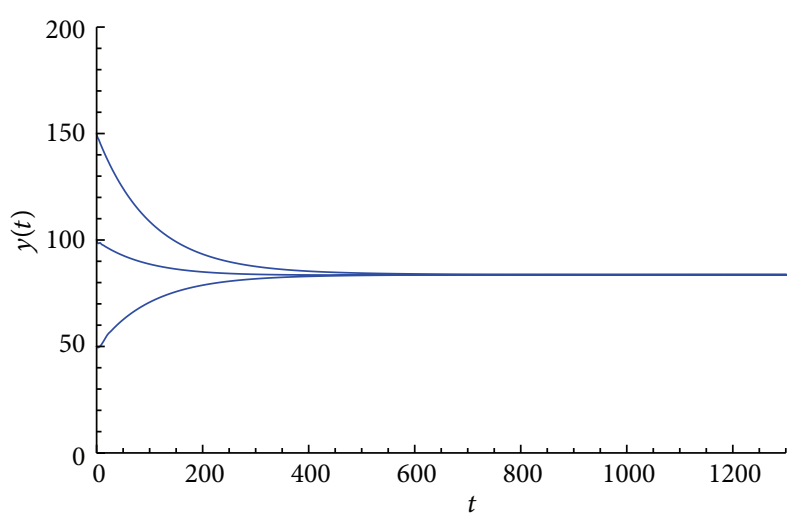

(b)

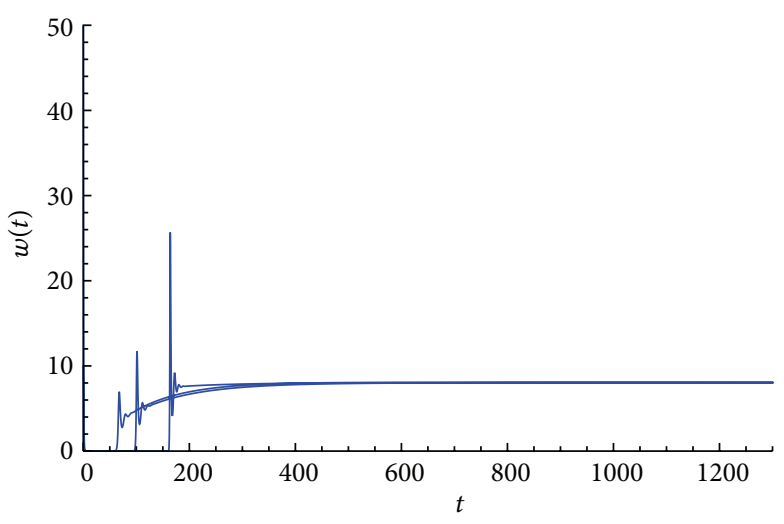

(d)

Figure 3: Trajectories of $x(t), y(t), v(t), w(t)$ for $\mathfrak{R}_{0}^{1}=2.15743$ and $\mathfrak{R}_{1}^{1}=2.05092$, and $E_{2}=(9818.03,83.7059,1933.33,8.0456)$ are globally asymptotically stable. $x(t), y(t), v(t)$, and $w(t)$ versus $t$ are illustrated by (a), (b), (c), and (d).

$$
\begin{aligned}
& +\frac{\beta e^{-m \tau} x v}{1+a x+b v}-\frac{\beta e^{-m \tau} x(t-\tau) v(t-\tau)}{1+a x(t-\tau)+b v(t-\tau)} \\
& +p y_{2} \ln \frac{x(t-\tau) v(t-\tau)}{x v} \frac{1+a x+b v}{1+a x(t-\tau)+b v(t-\tau)} \\
& =-\frac{d\left(1+b v_{2}\right) e^{-m \tau}}{x\left(1+a x_{2}+b v_{2}\right)}\left(x-x_{2}\right)^{2} \\
& +p y_{2}\left[1-\frac{x_{2}\left(1+a x+b v_{2}\right)}{x\left(1+a x_{2}+b v_{2}\right)}+\ln \frac{x_{2}\left(1+a x+b v_{2}\right)}{x\left(1+a x_{2}+b v_{2}\right)}\right] \\
& +p y_{2}\left[1-\frac{y_{2}\left(1+a x_{2}+b v_{2}\right) x(t-\tau) v(t-\tau)}{y x_{2} v_{2}(1+a x(t-\tau)+b v(t-\tau))}\right. \\
& \left.+\ln \frac{y_{2}\left(1+a x_{2}+b v_{2}\right) x(t-\tau) v(t-\tau)}{y x_{2} v_{2}(1+a x(t-\tau)+b v(t-\tau))}\right] \\
& -\frac{p y_{2} b(1+a x)\left(v-v_{2}\right)^{2}}{v_{2}(1+a x+b v)\left(1+a x+b v_{2}\right)}
\end{aligned}
$$$$
+p y_{2}\left[1-\frac{v_{2} y}{y_{2} v}+\ln \frac{v_{2} y}{y_{2} v}\right]
$$

Therefore, $U_{2}^{\prime} \leq 0$ holds for all $x, y, v, w>0$. Thus, the endemic equilibrium $E_{2}$ is stable. And we have $U_{2}^{\prime}=0$ if and only if $x=x_{2}, y=y_{2}, v=v_{2}$, and $w=w_{2}$ hold. The largest compact invariant set in $\left\{(x, y, v, w) \mid U_{2}^{\prime}=0\right\}$ is the singleton $\left\{E_{2}\right\}$. Therefore, the endemic equilibrium $E_{2}$ is globally asymptotically stable by the LaSalle invariance principle when $\mathfrak{R}_{1}^{1}>1$. This completes the proof.

\section{Numerical Example}

We now carry out some numerical simulations to support our theoretic results for the dynamics of the concentration of uninfected target cells $x(t)$ [cells $\mathrm{mL}^{-1}$ ], productively infected cells $y(t)$ [cells $\mathrm{mL}^{-1}$ ], free virus in the serum $v(t)$ [virion cells $\left.\mathrm{mL}^{-1}\right]$ antibodies $w(t)[\mu \mathrm{g}]$. We set $m=0$ and $\tau=0.5$ for the sake of simplification. The values of the parameters $\lambda, d, \beta$, 
$p, k, \mu, q, g$, and $h$ are found in Pawelek et al. [22] and Wang et al. [23].

Parameters in Figure 1 are $\lambda=46$ cells $\mathrm{mL}^{-1}, d=$ 0.0046 day $^{-1}, \beta=4.8 \times 10^{-7} \mathrm{~mL}$ virion-day ${ }^{-1}, a=0.01$, $b=0.005, p=0.01$ day $^{-1}, k=11.349$ day $^{-1}, \mu=0.25$ day $^{-1}$, $q=0.03, g=0.0015$, and $h=2.9 \mathrm{day}^{-1}$. In this case, we obtain $\mathfrak{R}_{0}^{1}=0.215743$ and $\mathfrak{R}_{1}^{1}=0.127203$. From (i) of Theorem $5, E_{0}=\left(10^{4}, 0,0,0\right)$ is globally asymptotically stable.

Parameters in Figure 2 are $\lambda=46$ cells $\mathrm{mL}^{-1}, d=$ 0.0046 day $^{-1}, \beta=2.4 \times 10^{-7} \mathrm{~mL}$ virion-day ${ }^{-1}, a=0.01$, $b=0.005, p=0.01$ day $^{-1}, k=11.349$ day $^{-1}, \mu=0.25$ day $^{-1}$, $q=0.03, g=0.0015$, and $h=2.9$ day $^{-1}$. In this case, we obtain $\mathfrak{R}_{0}^{1}=1.07872$ and $\mathfrak{R}_{1}^{1}=0.982187$. From (ii) of Theorem $5, E_{1}=(9924.5,34.7292,1576.57,0)$ is globally asymptotically stable.

Parameters in Figure 3 are $\lambda=46$ cells $\mathrm{mL}^{-1}, d=$ 0.0046 day $^{-1}, \beta=4.8 \times 10^{-6} \mathrm{~mL}$ virion-day ${ }^{-1}, a=0.01$, $b=0.005, p=0.01$ day $^{-1}, k=11.349$ day $^{-1}, \mu=0.25$ day $^{-1}$, $q=0.03, g=0.0015$, and $h=2.9$ day $^{-1}$. In this case, we obtain $\mathfrak{R}_{0}^{1}=2.15743$ and $\Re_{1}^{1}=2.05092$. From (iii) of Theorem $5, E_{2}=(9818.03,83.7059,1933.33,8.0456)$ is globally asymptotically stable.

\section{Conclusions and Discussion}

From Theorem 2 we can see that

$$
\begin{aligned}
v^{1}-v^{*}= & \sum_{i=1}^{n} \frac{k_{i}}{\mu} \frac{\sum_{i=1}^{n} \beta_{i} e^{-m_{i} \tau} k_{i} \lambda_{i}-d_{i} p_{i} \mu-a_{i} p_{i} \mu \lambda_{i}}{\sum_{i=1}^{n} p_{i} k_{i} d_{i} b_{i}+\beta_{i} k_{i} p_{i}-a_{i} p_{i}^{2} \mu e^{m_{i} \tau}} \\
& \times\left(\Re_{1}-1\right)>0,
\end{aligned}
$$

so the antibody immune response has a positive role in reduction of the virus. Huang et al. [7] investigated the global dynamics of basic in-host dynamics model with BeddingtonDeAngelis infection rate. But their models did not conclude immune response and intracellular delay. On the other hand, we improve the results in [8] from bilinear incidence rate to Beddington-DeAngelis incidence rate. It is similar to the well-known Holling type II functional response but has an extra term $b v$ in the denominator which models mutual interference between viruses. When $a>0, b=0$, the Beddington-DeAngelis functional response is simplified to Holling type II functional response [21]. When $a=0, b>0$, it expresses a saturation response; our model will reduce to the model studied in [9].

\section{Conflict of Interests}

The authors declare that there is no conflict of interests regarding the publication of this paper.

\section{Acknowledgments}

The authors would like to thank the anonymous referees and the editor for their very helpful suggestions and comments which led to improvements of their original paper.
X. Tian is supported by Project of Graduate-Student Innovation Study of Heilongjiang University (no. YJSCX2014068HLJU). J. Wang is supported by National Natural Science Foundation of China (nos. 11401182 and 11471089), Natural Science Foundation of Heilongjiang Province (no. A201415), Science and Technology Innovation Team in Higher Education Institutions of Heilongjiang Province (no. 2014TD005), Project funded by China Postdoctoral Science Foundation (no. 2014M552295), and Project funded by Chongqing Postdoctoral Foundation (no. Xm2014024).

\section{References}

[1] C. Chiyaka, W. Garira, and S. Dube, "Modelling immune response and drug therapy in human malaria infection," Computational and Mathematical Methods in Medicine, vol. 9, no. 2, pp. 143-163, 2008.

[2] A. S. Perelson, "Modelling viral and immune system dynamics," Nature Reviews Immunology, vol. 2, no. 1, pp. 28-36, 2002.

[3] T. Kajiwara and T. Sasaki, "A note on the stability analysis of pathogen-immune interaction dynamics," Discrete and Continuous Dynamical Systems Series B, vol. 4, no. 3, pp. 615-622, 2004.

[4] A. Korobeinikov, "Global properties of basic virus dynamics models," Bulletin of Mathematical Biology, vol. 66, no. 4, pp. 879-883, 2004.

[5] C. C. McCluskey, "Complete global stability for an SIR epidemic model with delay-distributed or discrete," Nonlinear Analysis: Real World Applications, vol. 11, no. 1, pp. 55-59, 2010.

[6] M. Y. Li and H. Shu, "Impact of intracellular delays and targetcell dynamics on in vivo viral infections," SIAM Journal on Applied Mathematics, vol. 70, no. 7, pp. 2434-2448, 2010.

[7] G. Huang, W. Ma, and Y. Takeuchi, "Global properties for virus dynamics model with Beddington-DeAngelis functional response," Applied Mathematics Letters, vol. 22, no. 11, pp. 1690$1693,2009$.

[8] S. Wang and D. Zou, "Global stability of in-host viral models with humoral immunity and intracellular delays," Applied Mathematical Modelling, vol. 36, no. 3, pp. 1313-1322, 2012.

[9] X. Wang and S. Liu, "A class of delayed viral models with saturation infection rate and immune response," Mathematical Methods in the Applied Sciences, vol. 36, no. 2, pp. 125-142, 2013.

[10] D. S. Callaway and A. S. Perelson, "HIV-1 infection and low steady state viral loads," Bulletin of Mathematical Biology, vol. 64, no. 1, pp. 29-64, 2002.

[11] A. S. Perelson and P. W. Nelson, "Mathematical analysis of HIV1 dynamics in vivo," SIAM Review, vol. 41, no. 1, pp. 3-44, 1999.

[12] A. M. Elaiw and X. Xia, "HIV dynamics: analysis and robust multirate MPC-based treatment schedules," Journal of Mathematical Analysis and Applications, vol. 359, no. 1, pp. 285-301, 2009.

[13] A. M. Elaiw, "Global properties of a class of HIV models," Nonlinear Analysis: Real World Applications, vol. 11, no. 4, pp. 2253-2263, 2010.

[14] A. M. Elaiw and M. A. Alghamdi, "Global properties of virus dynamics models with multitarget cells and discrete-time delays," Discrete Dynamics in Nature and Society, vol. 2011, Article ID 201274, 19 pages, 2011.

[15] A. M. Elaiw, "Global properties of a class of virus infection models with multitarget cells," Nonlinear Dynamics, vol. 69, no. 1-2, pp. 423-435, 2012. 
[16] A. M. Elaiw and S. A. Azoz, "Global properties of a class of HIV infection models with Beddington-DeAngelis functional response," Mathematical Methods in the Applied Sciences, vol. 36, no. 4, pp. 383-394, 2013.

[17] A. Elaiw, I. Hassanien, and S. Azoz, "Global stability of HIV infection models with intracellular delays," Journal of the Korean Mathematical Society, vol. 49, no. 4, pp. 779-794, 2012.

[18] J. Wang and L. Guan, "Global stability for a HIV-1 infection model with cell-mediated immune response and intracellular delay," Discrete and Continuous Dynamical Systems B, vol. 17, no. 1, pp. 297-302, 2012.

[19] J. Wang, G. Huang, and Y. Takeuchi, "Global asymptotic stability for HIV-1 dynamics with two distributed delays," Mathematical Medicine and Biology, vol. 29, no. 3, pp. 283-300, 2012.

[20] J. Wang, G. Huang, Y. Takeuchi, and S. Liu, "Sveir epidemiological model with varying infectivity and distributed delays," Mathematical Biosciences and Engineering, vol. 8, no. 3, pp. 875888, 2011.

[21] D. Li and W. Ma, "Asymptotic properties of a HIV-1 infection model with time delay," Journal of Mathematical Analysis and Applications, vol. 335, no. 1, pp. 683-691, 2007.

[22] K. A. Pawelek, S. Liu, F. Pahlevani, and L. Rong, "A model of HIV-1 infection with two time delays: mathematical analysis and comparison with patient data," Mathematical Biosciences, vol. 235, no. 1, pp. 98-109, 2012.

[23] J. Wang, J. Pang, T. Kuniya, and Y. Enatsu, "Global threshold dynamics in a five-dimensional virus model with cell-mediated, humoral immune responses and distributed delays," Applied Mathematics and Computation, vol. 241, pp. 298-316, 2014. 


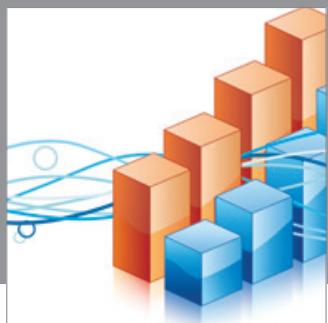

Advances in

Operations Research

mansans

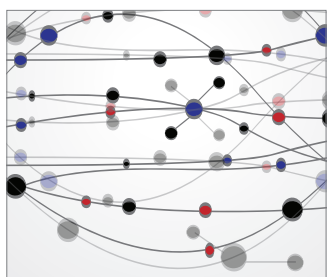

The Scientific World Journal
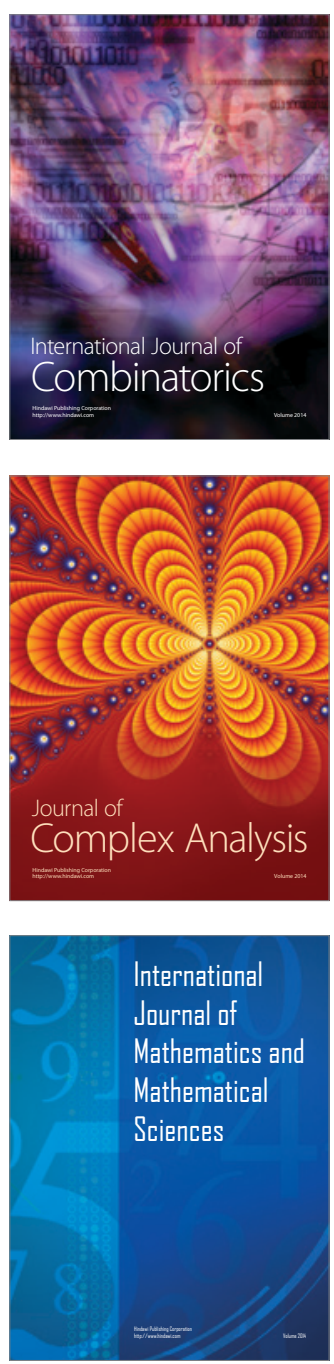
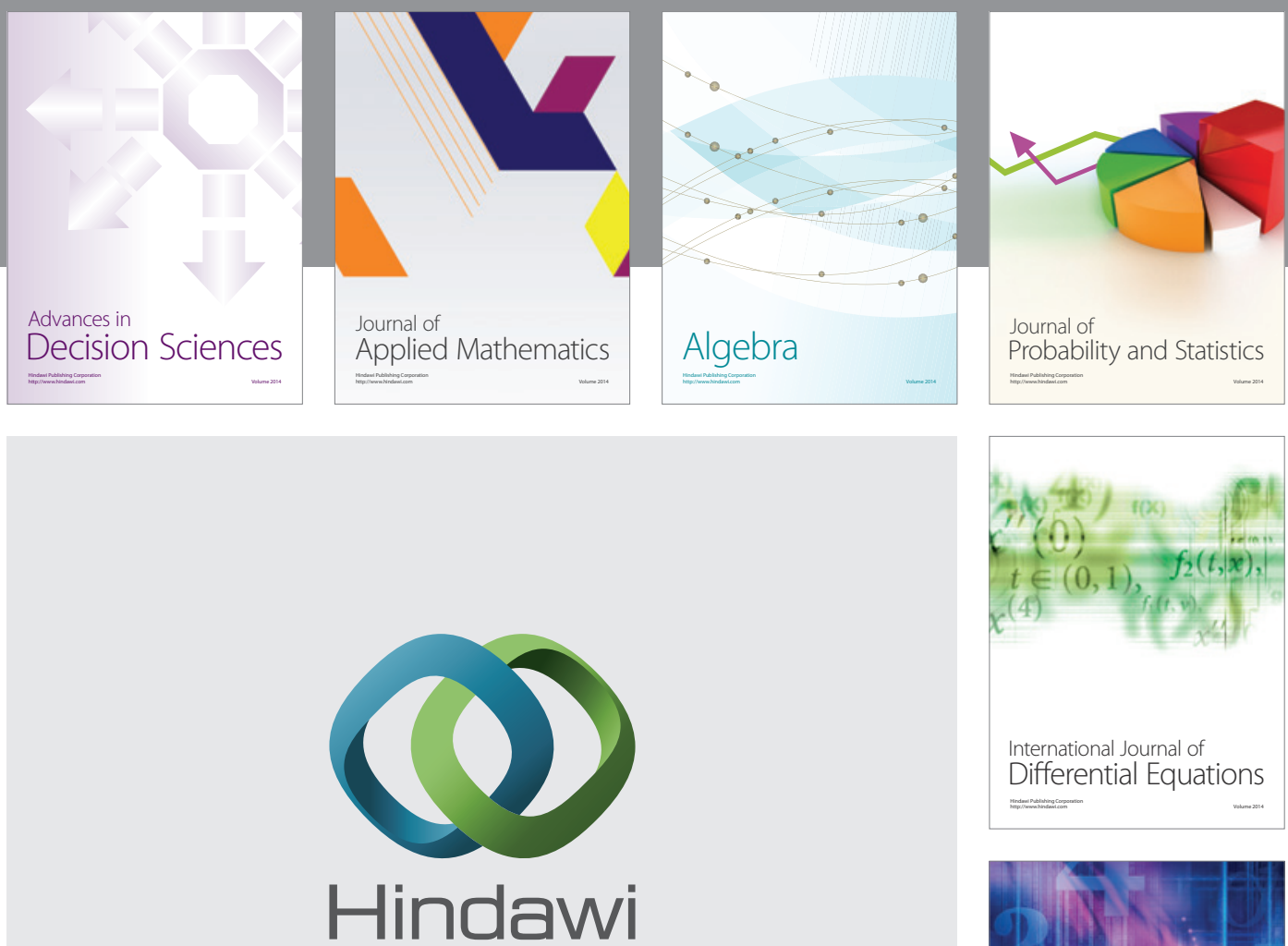

Submit your manuscripts at http://www.hindawi.com
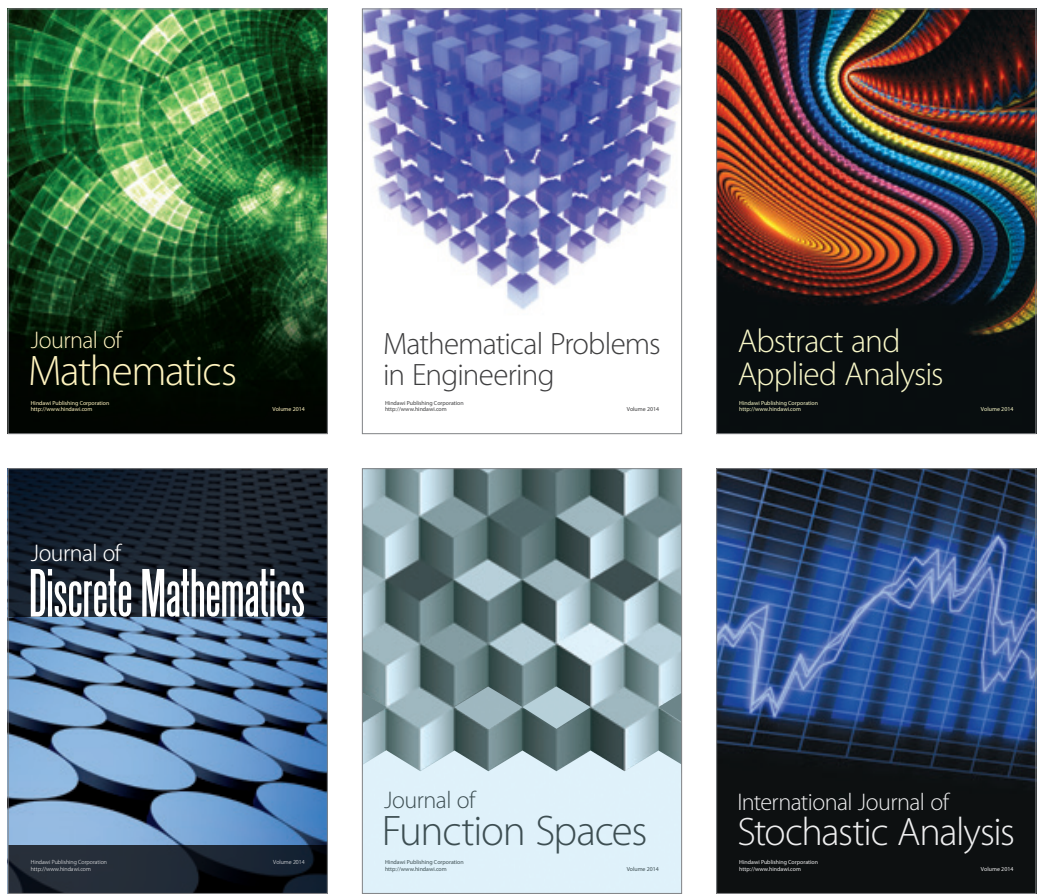

Journal of

Function Spaces

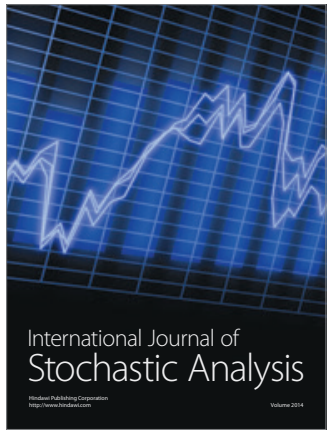

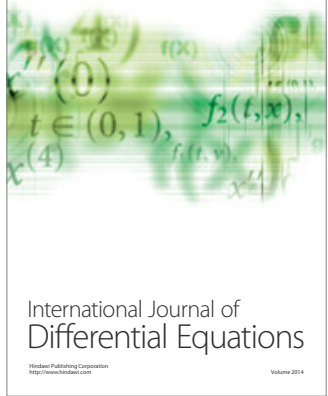
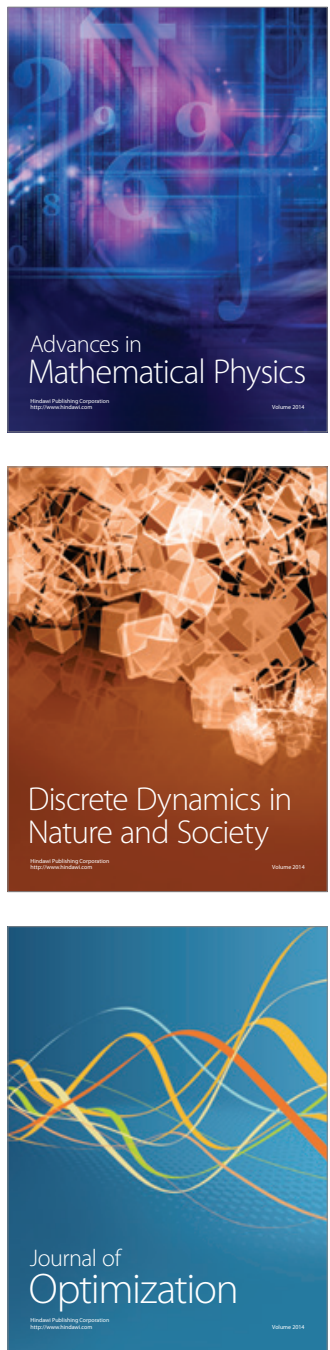